\title{
Reliability of Clinical Assessment Methods to Measure Scapular Upward Rotation: A Critically Appraised Topic
}

\author{
Oliver Silverson, Nicole Cascia, Carolyn M. Hettrich, Matt Hoch, and Tim Uhl
}

\begin{abstract}
Clinical Scenario: Assessing movement of the scapula is an important component in the evaluation and treatment of the shoulder complex. Currently, gold-standard methods to quantify scapular movement include invasive technique, radiation, and 3D motion systems. This critically appraised topic focuses on several clinical assessment methods of quantifying scapular upward rotation with respect to their reliability and clinical utility. Clinical Question: Is there evidence for noninvasive methods that reliably assess clinical measures of scapular upward rotation in subjects with or without shoulder pathologies? Summary of Key Findings: Four studies were selected to be critically appraised. The quality appraisal of diagnostic reliability checklist was used to score the articles on methodology and consistency. Three of the 4 studies demonstrated support for the clinical question. Clinical Bottom Line: There is moderate evidence to support reliable clinical methods for measuring scapular upward rotation in subjects with or without shoulder pathology. Strength of Recommendation: There is moderate evidence to suggest there are reliable clinical measures to quantify scapular upward rotation in patients with or without shoulder pathology.
\end{abstract}

Keywords: scapula, CAT, clinical assessment

\section{Clinical Scenario}

Quantifying movement of the scapula is an important component in the evaluation and treatment of the shoulder complex due to its role in scapulohumeral rhythm during overhead motion. ${ }^{1-3}$ Scapular upward rotation has been identified to be an essential component of glenohumeral elevation. ${ }^{4}$ Restrictions in the scapular upward rotation have been linked to pathologies such as impingement, instability, and tendinopathies. ${ }^{4,5}$ Challenges clinicians face when measuring scapular motion include the deep position of the scapula under the overlaying musculature and soft tissue, and along with its multidirectional axis of rotation. ${ }^{1,6,7}$ To accurately quantify scapular movement, fluoroscopy, and intracortical pinning have been utilized as the gold-standard methods of scapular assessment. ${ }^{8,9}$ However, due to the obstacles behind radiography and 3D instrumentation, such as radiation, invasive technique, cost for equipment, and time investment, it has become apparent that there is a clinical need for a reliable and valid noninvasive method to measure scapular kinematics. ${ }^{10,11}$ In the literature, different assessment methods have been reported, focusing on the reliability of each respective method. In this critically appraisal topic (CAT), several methods of quantifying scapular kinematics are appraised for their respective reliability and clinical utility of each method designed to measure scapular upward rotation.

\section{Focused Clinical Question}

Is there evidence for noninvasive methods that reliably assess clinical measures of scapular upward rotation in subjects with or without shoulder pathologies?

Silverson is with the Division of Athletic Training, Department of Rehabilitation Sciences, College of Health Sciences, University of Kentucky, Lexington, KY. Cascia, Hoch, and Uhl are with the Department of Rehabilitation Sciences, University of Kentucky, Lexington, KY. Hettrich is with the Department of Orthopaedic Surgery, University of Kentucky, Lexington, KY. Silverson (Oliver. Silverson@uky.edu) is corresponding author.

\section{Summary of Search}

- The initial literature search using the Boolean Operators: Term (TX) scapular rotation, OR TX scapular motion, AND TX upward rotation, AND TX measurement, AND TX reliability for articles published since 2007 produced 678 results (Table 1).

- The second set of Boolean Operators was applied in addition to the original literature search that included: NOT TX stroke, NOT TX Palsy, published since 2010 and returned 535 results.

- The 535 results from the refined literature search were reviewed for title and abstract content relevant to the clinical question.

- Forty-six articles were identified for further review of content and methodology. Articles from the reference lists from the 46 articles were also considered. Duplicate articles were removed.

- Twenty-five articles were eliminated based on the exclusion criteria. The remaining 21 studies were screened for methodology and were selected for further appraisal after general clinical applicability had been determined for the assessment method. Each article was evaluated for original research and reports of the reliability of an independent clinical assessment method.

- Four articles were chosen to be included in the CAT. 10,12-14 The articles completed by Johnson et $\mathrm{al}^{13}$ and Watson et $\mathrm{al}^{10}$ were selected from the reference lists of Tucker et $\mathrm{al}^{12}$ and De Groef et al, ${ }^{14}$ respectively.

\section{Evidence Appraisal}

- Each study reported reliability values for the assessment method examined in the form of the intraclass correlation coefficient (ICC) values for intrarater, interrater, or both. ICC values are presented in Table 2. The ICC values of an assessment method were examined for the strength of reliability with values less than 0.75 being poor to moderate and values above 0.75 representing good to excellent reliability. ${ }^{15}$ 
Table 1 Flowchart of Search

\begin{tabular}{|c|c|}
\hline $\begin{array}{l}\text { Studies } \\
\text { retrieved } \\
\mathrm{N}=678\end{array}$ & $\begin{array}{l}\text { - Database search: EBSCOhost (CINAHL, CINAHL with full text, MEDLINE, and SPORTDiscus). } \\
\text { - Boolean operators: TX scapular rotation, OR TX scapular motion, AND TX upward rotation, AND TX measurement, AND TX } \\
\text { reliability, published since 2007. Duplicates removed. }\end{array}$ \\
\hline $\begin{array}{l}\text { Studies } \\
\text { retrieved } \\
\mathrm{N}=535\end{array}$ & - EBSCOhost search with the addition of Boolean operators: NOT TX stroke, NOT TX Palsy, published since 2010. \\
\hline $\begin{array}{l}\text { Studies } \\
\text { reviewed } \\
\mathrm{N}=46\end{array}$ & - Relevant studies and reference lists were reviewed for title and abstract content. \\
\hline $\begin{array}{l}\text { Studies } \\
\text { excluded } \\
\mathrm{N}=25\end{array}$ & $\begin{array}{l}\text { - Studies reporting on 3D instrumentation (16), scapular rehabilitation programs (5), glenohumeral assessment motion only (2), and } \\
\text { validity only (2). }\end{array}$ \\
\hline $\begin{array}{l}\text { Studies } \\
\text { reviewed } \\
\mathrm{N}=21\end{array}$ & - Studies examined for original research, methodology, and reports of reliability. \\
\hline $\begin{array}{l}\text { Studies } \\
\text { included } \\
\mathrm{N}=4\end{array}$ & $\begin{array}{l}\text { - Articles included in the critically appraisal topic. } \\
\text { - Original search (2): De Groef et } \mathrm{al}^{14} \text { and Tucker et al. } \\
\text { - Reference lists of included articles (2): Johnson et } \mathrm{al}^{13} \text { (Tucker et } \mathrm{al}^{12} \text { ) and Watson et al }{ }^{10} \text { (De Groef et } \mathrm{al}^{14} \text { ). }\end{array}$ \\
\hline
\end{tabular}

- The quality appraisal of diagnostic reliability (QAREL) checklist was used by a single reviewer to critically appraise the 4 studies. The QAREL evaluates studies for diagnostic reliability through methodology and consistency. ${ }^{16}$ A study receiving a high score is likely to satisfy a higher number of items on the checklist, indicating that the study reported on the specific methodology in the study design. ${ }^{16}$

- The level of evidence was determined using an adaptation of the system described by van Tulder et al. ${ }^{17}$ The systematic approach indicates that studies with higher methodological consistency, determined by the QAREL, signify a higher level of evidence. ${ }^{17}$

- A strong level of evidence was designated for an assessment method receiving above $80 \%$ on the QAREL, a moderate level of evidence was given to an assessment method receiving from $50 \%$ to $79 \%$, and a limited level of evidence was assigned to an assessment method receiving below $50 \%$ on the QAREL.

\section{Key Findings}

- All studies included in the CAT sought to quantify scapular upward rotation using a noninvasive and clinically applicable method. The studies conducted by Johnson et $\mathrm{al}^{13}$ and Tucker et $\mathrm{al}^{12}$ determined good to excellent intrarater reliability (ICC 0.89-0.96, 0.89-0.97, respectively) for the method of assessment based on a modified digital protractor, called an inclinometer. The other assessment method investigated in this CAT was a 2 gravity referenced inclinometer system, found to have good to excellent intrarater reliability (ICC 0.88-0.94) by Watson et al, ${ }^{10}$ but weak interrater reliability (ICC 0.14-0.52) by De Groef et al. ${ }^{14}$

- QAREL scores on the 4 studies ranged from $50 \%$ to $87 \%$. Limited to moderate levels of evidence were found to exist across the 4 studies included in the CAT. The individual scores for each study are displayed in Table 2.

- Three of the 4 studies appraised demonstrated support for the clinical question (Johnson et al, ${ }^{13}$ Tucker et al, ${ }^{12}$ and Watson et $\mathrm{al}^{10}$ ), whereas 1 study did not (De Groef et $\mathrm{al}^{14}$ ).
- A moderate level of evidence was determined in support of the clinical question. This was concluded with consideration of the QAREL scores of the 3 studies that exhibited support and the 1 opposing study. The moderate QAREL average of the 3 studies in support (60\%) versus the strong QAREL score $(87 \%)$ against indicated the level of evidence was restricted to moderate.

\section{Clinical Bottom Line}

There is moderate evidence to support reliable clinical methods for measuring scapular upward rotation exist when limited to a single session for the assessment of subjects with or without shoulder pathology. The clinical methods examined were found to be reliable when used by a single rater. Future research should aim to establish between-rater and between-session reliability.

\section{Strength of Recommendation}

There is moderate evidence to suggest there are reliable clinical measures to quantify scapular upward rotation in patients with or without shoulder pathology. The systematic approach described by van Tulder et $\mathrm{al}^{17}$ used to determine the moderate level of evidence is based upon an article's support for the clinical question and the methodological quality of the study.

\section{Search Strategy}

\section{Terms Used to Guide Search Strategy}

- Patient: subjects with or without shoulder pathologies

- Intervention: intrarater/interrater reliability

- Comparison: none

- Outcome: scapular upward rotation

\section{Sources of Evidence Searched}

EBSCOhost: CINAHL, CINAHL with full text, MEDLINE, SPORTDiscus, and additional resources obtained via review of reference lists. 


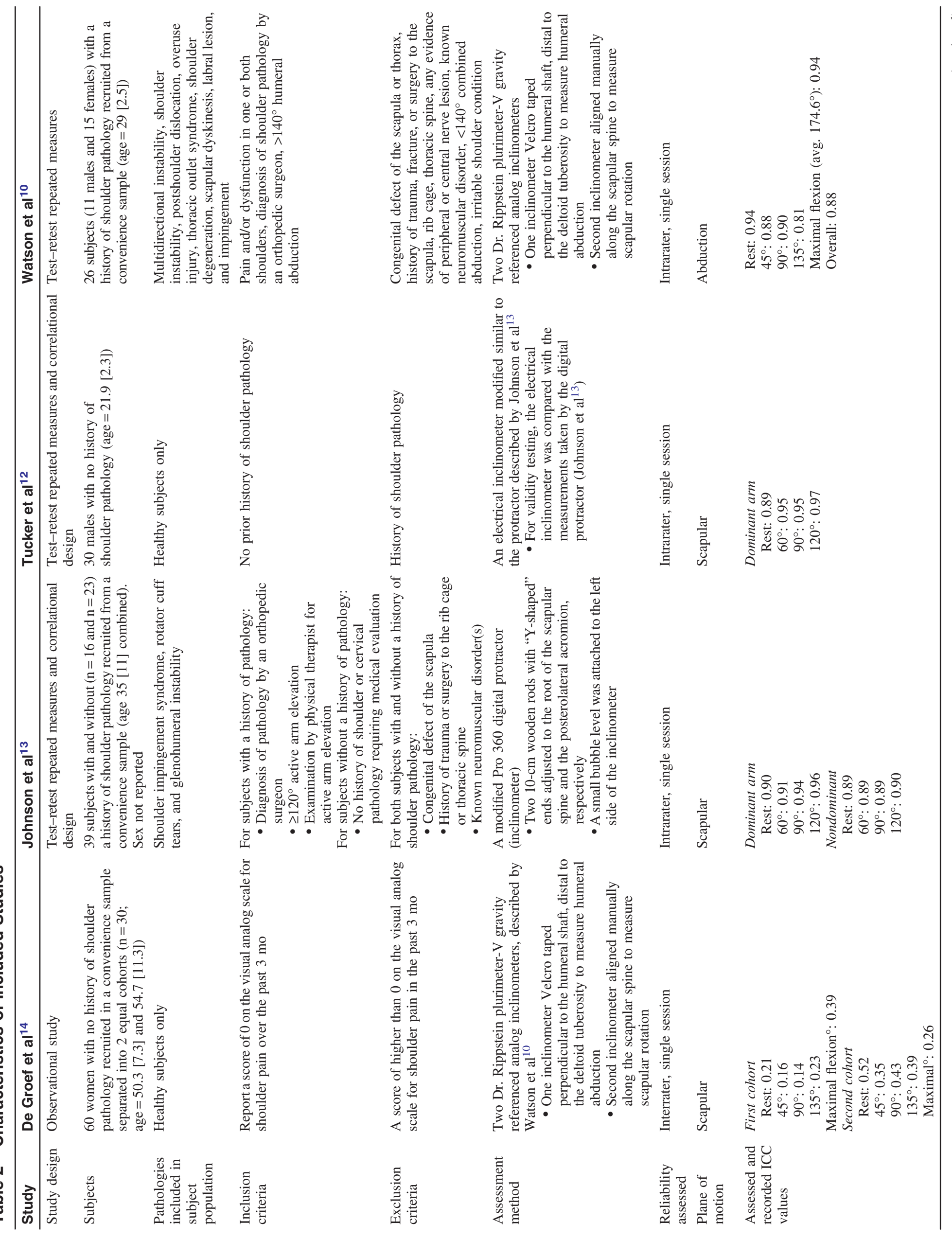




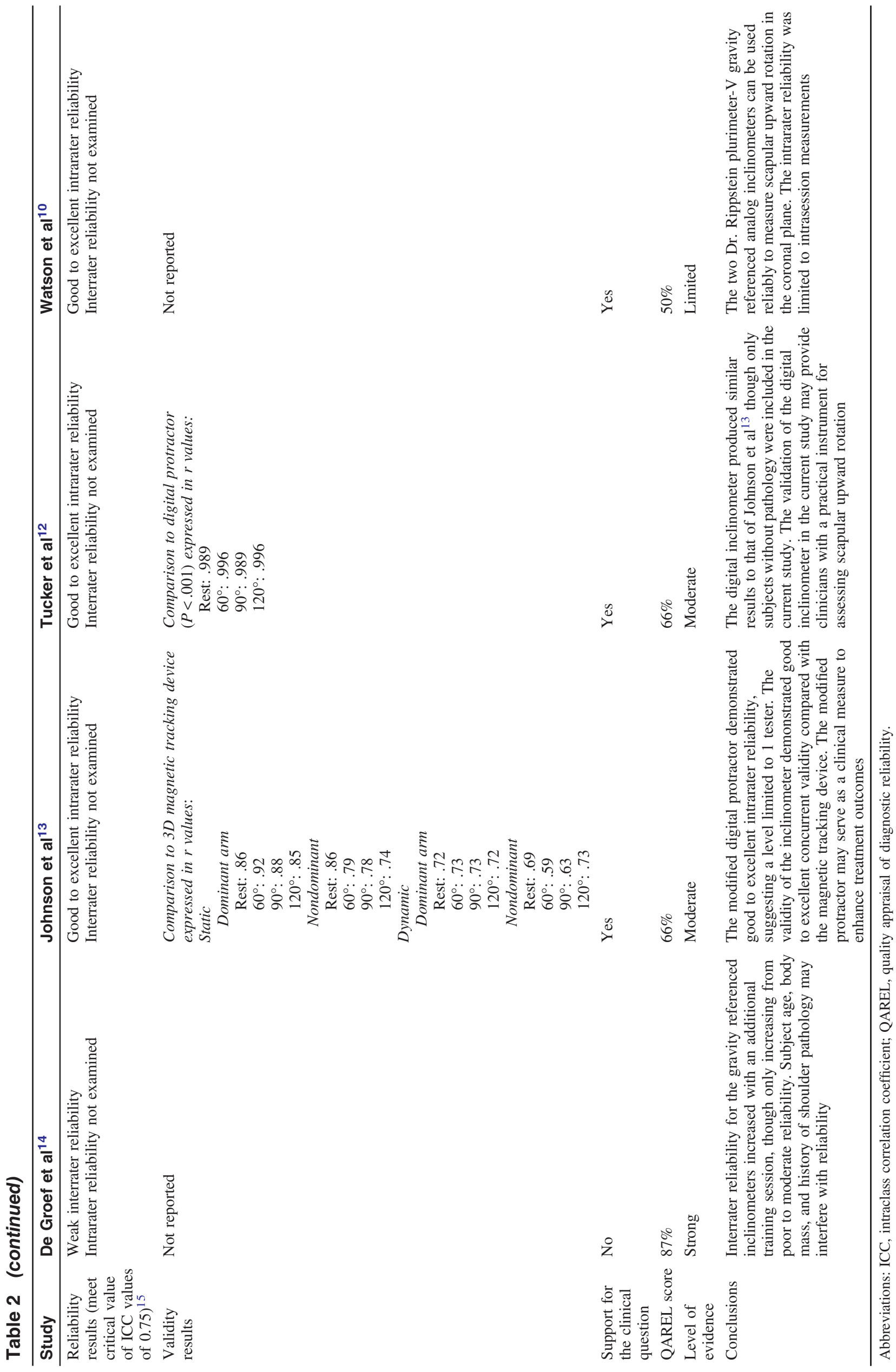




\section{Inclusion and Exclusion Criteria}

\section{Inclusion Criteria}

\section{Studies that}

- Included a clinical assessment tool for measuring scapular upward rotation

- Reported intrarater/interrater reliability values for the assessment method

- Reported on patients with or without shoulder pathology

- Used subjects 18 years of age or older

- Reported in English

\section{Exclusion Criteria}

Studies that

- Examined assessment methods requiring software or computer analysis

- Involved invasive procedures as a means to measure scapular movement

- Used 3D motion analysis methods as the only method of evaluation

- Did not include reliability as an outcome of the study

- Included stroke or palsy patients

- Reported on cadaveric measurements

- Abstract only

- Not written in English

\section{Results of Search}

Four relevant studies were located and categorized as shown in Table 3.

\section{Summary of Best Evidence}

The summary characteristics of the four articles that were included in this study are depicted in Table 2.

\section{Implications for Practice, Education, and Future Research}

The current literature suggests that there are reliable noninvasive clinical assessment methods to measure scapular upward rotation in subjects with or without shoulder pathology. Inclinometer-based assessment performed by a single rater demonstrated high insession reliability reported by Johnson et al, ${ }^{13}$ Tucker et al, ${ }^{12}$ and Watson et al. ${ }^{10}$ The ICC values for each method are presented in Table 2, values above 0.75 were considered good to excellent reliability for the assessment method being tested. ${ }^{15}$

The modified electrical inclinometer (Noraxon USA Inc, Scottsdale, AZ) investigated by Tucker et $\mathrm{al}^{12}$ was found to have the highest intrarater reliability (ICC 0.89-0.97) among all of the methods assessed in the CAT. The study also reported on the validity of the electrical inclinometer by comparing to measurements recorded by the modified digital protractor (Pro 360; MacklanburgDuncan, Oklahoma City, OK) as described by Johnson et al. ${ }^{13}$ The correlation of the electrical inclinometer's static scapular measurements to those taken from the digital protractor was found to have excellent validity ( $r$ values .989-.996, $P<.001)$ in each of the scapular positions tested, presented in Table 2 . These high values demonstrate the electrical inclinometer investigated by Tucker et $\mathrm{al}^{12}$ to be a reliable method for quantifying scapular upward rotation.

Although the purpose of the study by Tucker et $\mathrm{al}^{12}$ was to establish reliability and validity of the electrical inclinometer, it simultaneously proposes between-session reliability of the modified digital protractor previously investigated by Johnson et $\mathrm{al}^{13}$ (intrarater ICC 0.89-0.96). The notion of between-session reliability is facilitated by the high correlative values ( $r$ values .989-.996, $P<.001)$ reported by Tucker et $\mathrm{al}^{12}$ when validating the electrical inclinometer to the modified digital protractor. Although the study by Tucker et $\mathrm{al}^{12}$ evaluated subjects with no history of shoulder pathology, the study by Johnson et $\mathrm{al}^{13}$ used subjects with and without shoulder pathology. The high correlative values on each respective subject population suggest that further investigation of between-session reliability on the modified digital protractor could result in good to excellent interrater reliability values as well.

A different form of inclinometer-based assessment investigated in the CAT is a 2 inclinometer system first investigated by Watson et $\mathrm{al}^{10}$ known as the "two Dr. Rippstein plurimeter-V gravity referenced analog inclinometer system." The 2 inclinometer system demonstrated excellent intrarater reliability (ICC 0.88-0.94) ${ }^{7}$ when implemented on subjects with a history of shoulder pathology but failed to replicate good reliability when examined by De Groef et $\mathrm{al}^{14}$ on healthy subjects. Poor intrarater reliability (ICC 0.14-0.39) found in the first cohort of 30 middle-aged women only slightly improved from poor to moderate in the second cohort (ICC 0.26 0.52 ) even after additional investigator training. ${ }^{14}$ The reduced replication of reliability for the 2 gravity referenced inclinometer system reported by De Groef et $\mathrm{al}^{14}$ suggests that the reliability of the system is limited to within session and to a single rater when used on subjects with a history of shoulder pathology.

This CAT does not report on studies without limitation. A difficulty that is unanimously described in the literature surrounding noninvasive techniques in quantifying scapular movement is a skin artifact. ${ }^{6,8,12,13}$ Furthermore, postural fatigue, breathing rhythm, and body size are also limitations that studies face during measurement recording and replication of scapular movement. ${ }^{11,12,14}$ The number of subjects in each study varied and consistency was lacking around the number of healthy patients versus those who had a history of

Table 3 Summary of Study Designs and Articles Retrieved

\begin{tabular}{lll}
\hline Level of evidence $^{\mathbf{a}}$ & Study design & Author \\
\hline Strong & Observational study & De Groef et al \\
Moderate & Test-retest repeated measures and correlational design & Johnson et al ${ }^{13}$ \\
Moderate & Test-retest repeated measures and correlational design & Tucker et al $^{12}$ \\
Limited & Test-retest repeated measures & Watson et al $^{10}$ \\
\hline
\end{tabular}

${ }^{a}$ Based on the systematic approach described by van Tulder et al. ${ }^{17}$ 
shoulder pathology. More investigation is needed to determine if there is reason to suspect the clinical assessment methods included in this CAT are a better fit for subjects with or without shoulder pathology. In addition, 2 studies examined static scapular measurements at rest, $60^{\circ}, 90^{\circ}$, and $120^{\circ}$ of scaption, whereas others investigated scapular position at rest, $45^{\circ}, 90^{\circ}$, and $135^{\circ}$ of glenohumeral elevation in scaption. ${ }^{10,12-14}$ With all limitations considered, the QAREL checklist was used as an appraisal tool to assess studies on methodological quality and consistency. The checklist examined the methods of each study through procedural consistencies such as position randomization and researcher blinding. ${ }^{16}$

Interestingly, the study by De Groef et $\mathrm{al}^{14}$ recorded the highest QAREL score (87\%) of all the other articles included in the CAT, thus providing a strong level of evidence arguing against the reliability of the previously reported clinical assessment method. ${ }^{10}$ The studies conducted by Johnson et $\mathrm{al}^{13}$ and Tucker et $\mathrm{al}^{12}$ each recorded equal QAREL scores of $66 \%$, indicating acceptable methodological quality. Combined with the high correlation values of the 2 inclinometer systems ( $r$ values .989-.996), the moderate level of evidence supports both the digital protractor and the electric inclinometer to be reliable clinical assessment methods of scapular upward rotation.

The information included in this CAT suggests that inclinometer-based assessment is a reliable method for measuring scapular upward rotation when used in a single session by a single rater. As a clinical tool, an inclinometer is a cost-effective method of motion assessment that can be easily implemented into a practice with little training. The ability to reliably quantify scapular upward rotation is a productive step in understanding scapular kinematics and the implications of deficits in scapular motion. Inclinometer-based assessment serves to provide clinicians with a reliable tool to measure scapular upward rotation without dedicating sizable amounts of clinical efforts.

Future research is needed to continue to understand the applicability of inclinometer-based assessment in clinical practice as an assessment tool for measuring scapular upward rotation. Between-session and between-rater reliability should be a focus of future studies as well as determining reliable measurements of dynamic scapular movement during clinical assessment. Lastly, validating a noninvasive scapular clinical assessment tool against a gold-standard device that demonstrates good criterion-validity would be valuable in helping accurately measure scapular motion. Acceptable assessment methods that carry criterion validity for scapular motion assessment include 3D electromagnetic motion capture systems, intracortical bone pins during dynamic movement, or fluoroscopy. ${ }^{11,18}$ Although there are many studies in the literature focusing on the validity of the scapular assessment, it is those studies that primarily focus on methods of 3D motion analysis rather than clinically applicable techniques. Therefore, establishing inclinometer-based assessment as a valid means of assessment would facilitate the clinical use of the method.

\section{References}

1. Terry GC, Chopp TM. Functional anatomy of the shoulder. J Athl Train. 2000;35(3):248-255. PubMed ID: 16558636

2. Kibler WB, Ludewig PM, McClure P, Uhl TL, Sciascia A. Scapular Summit 2009: introduction. July 16, 2009, Lexington, Kentucky. J Orthop Sports Phys Ther. 2009;39(11):A1-A13. PubMed ID: 19881011 doi:10.2519/jospt.2009.0303

3. Halder AM, Itoi E, An K. Anatomy and biomechanics of the shoulder. Orthop Clin North Am. 2000;31(2):159-176. PubMed ID: 10736387 doi:10.1016/S0030-5898(05)70138-3
4. Kibler WB, Ludewig PM, McClure PW, Michener LA, Bak K, Sciascia AD. Clinical implications of scapular dyskinesis in shoulder injury: the 2013 consensus statement from the 'Scapular Summit'. Br J Sports Med. 2013;47(14):877-885. PubMed ID: 23580420 doi:10.1136/bjsports-2013-092425

5. Kibler WB, Sciascia A. Current concepts: scapular dyskinesis. $\mathrm{Br} J$ Sports Med. 2010;44(5):300-305. PubMed ID: 19996329 doi:10. 1136/bjsm.2009.058834

6. Warner MB, Chappell PH, Stokes MJ. Measurement of dynamic scapular kinematics using an acromion marker cluster to minimize skin movement artifact. J Vis Exp. 2015(96):e51717. PubMed ID: 25742242 doi: $10.3791 / 51717$

7. McClure P, Tate AR, Kareha S, Irwin D, Zlupko E. A clinical method for identifying scapular dyskinesis, part 1: reliability. J Athl Train. 2009;44(2):160-164. PubMed ID: 19295960 doi:10.4085/10626050-44.2.160

8. Lempereur M, Brochard S, Leboeuf F, Remy-Neris O. Validity and reliability of 3D marker based scapular motion analysis: a systematic review. J Biomech. 2014;47:2219-2230. PubMed ID: 24856913 doi:10.1016/j.jbiomech.2014.04.028

9. Ludewig PM, Phadke V, Braman JP, Hassett DR, Cieminski CJ, LaPrade RF. Motion of the shoulder complex during multiplanar humeral elevation. J Bone Joint Surg Am. 2009;91(2):378-389. PubMed ID: 19181982 doi:10.2106/JBJS.G.01483

10. Watson L, Balster SM, Finch C, Dalziel R. Measurement of scapula upward rotation: a reliable clinical procedure. Br J Sports Med. 2005; 39(9):599-603. PubMed ID: 16118295 doi:10.1136/bjsm.2004. 013243

11. Larsen CM, Juul-Kristensen B, Lund H, Søgaard K. Measurement properties of existing clinical assessment methods evaluating scapular positioning and function. A systematic review. Physiother Theory Pract. 2014;30(7):453-482. PubMed ID: 24678755 doi:10.3109/ 09593985.2014.899414

12. Steven Tucker W, Lyndsey Ingram R. Reliability and validity of measuring scapular upward rotation using an electrical inclinometer. $J$ Electromyogr Kinesiol. 2012;22(3):419-423. PubMed ID: 22425395 doi:10.1016/j.jelekin.2012.02.013

13. Johnson MP, McClure PW, Karduna AR. New method to assess scapular upward rotation in subjects with shoulder pathology. J Orthop Sports Phys Ther. 2001;31(2):81-89. PubMed ID: 11232742 doi:10.2519/jospt.2001.31.2.81

14. De Groef A, Van Kampen M, Vervloesem N, et al. Inter-rater reliability of shoulder measurements in middle-aged women. Physiotherapy. 2017;103(2):222-230. PubMed ID: 27908458 doi:10. 1016/j.physio.2016.07.002

15. Portney LG, Watkins M. Foundations of Clinical Research: Applications to Practice. 3rd ed. Upper Saddle River, NJ: Pearson Prentice Hall; 2009.

16. Lucas NP, Macaskill P, Irwig L, Bogduk N. The development of a quality appraisal tool for studies of diagnostic reliability (QAREL). J Clin Epidemiol. 2010;63(8):854-861. PubMed ID: 20056381 doi: 10.1016/j.jclinepi.2009.10.002

17. van Tulder M, Furlan A, Bombardier C, Bouter L. Editorial Board of the Cochrane Collaboration Back Review Group. Updated method guidelines for systematic reviews in the Cochrane collaboration back review group. Spine. 2003;28(12):1290-1299. PubMed ID: 12811274 doi:10.1097/01.BRS.0000065484.95996.AF

18. Chu Y, Akins J, Lovalekar M, Tashman S, Lephart S, Sell T. Validation of a video-based motion analysis technique in 3-D dynamic scapular kinematic measurements. J Biomech. 2012;45(14): 2462-2466. PubMed ID: 22898626 doi:10.1016/j.jbiomech.2012. 06.025 